\title{
Nucleation and growth of GaN nanorods on Si (111) surfaces by plasma-assisted molecular beam epitaxy - The influence of Si- and Mg-doping
}

\author{
Florian Furtmayr, ${ }^{1}$ Martin Vielemeyer, ${ }^{1}$ Martin Stutzmann, ${ }^{1}$ Jordi Arbiol, ${ }^{2,3}$ Sònia Estradé, ${ }^{2}$ \\ Francesca Peirò, ${ }^{2}$ Joan Ramon Morante, ${ }^{2}$ and Martin Eickhoff ${ }^{1, a)}$ \\ ${ }^{1}$ Walter Schottky Institut, Technische Universität München, Am Coulombwall 3, 85748 Garching, Germany \\ ${ }^{2}$ EME/CeRMAE/IN ${ }^{2} U B$, Departament d'Electrònica, Universitat de Barcelona, c/ Marti Franquès 1, \\ E-08080 Barcelona, CAT, Spain \\ ${ }^{3}$ TEM-MAT, Serveis Cientificotécnics, Universitat de Barcelona, c/ Lluis Sole i Sabaris 1-3, \\ E-08080 Barcelona, CAT, Spain
}

(Received 11 February 2008; accepted 7 May 2008; published online 8 August 2008)

\begin{abstract}
The self-assembled growth of GaN nanorods on $\mathrm{Si}$ (111) substrates by plasma-assisted molecular beam epitaxy under nitrogen-rich conditions is investigated. An amorphous silicon nitride layer is formed in the initial stage of growth that prevents the formation of a $\mathrm{GaN}$ wetting layer. The nucleation time was found to be strongly influenced by the substrate temperature and was more than $30 \mathrm{~min}$ for the applied growth conditions. The observed tapering and reduced length of silicon-doped nanorods is explained by enhanced nucleation on nonpolar facets and proves Ga-adatom diffusion on nanorod sidewalls as one contribution to the axial growth. The presence of $\mathrm{Mg}$ leads to an increased radial growth rate with a simultaneous decrease of the nanorod length and reduces the nucleation time for high $\mathrm{Mg}$ concentrations. (C) 2008 American Institute of Physics.
\end{abstract}

[DOI: $10.1063 / 1.2953087$ ]

\section{INTRODUCTION}

Quasi-one-dimensional GaN nanowires or nanorods (NRs) have recently become a topic of intense research interest. Due to their low density of structural defects, they present a promising approach for the realization of improved optoelectronic or electronic devices. ${ }^{1-3}$ The growth of GaN NRs by plasma-assisted molecular beam epitaxy (PAMBE) has been reported on different substrate materials, including $\mathrm{Si}$ (111), Si (001), or sapphire, ${ }^{4,5}$ as well as on GaN or AlN wetting layers. ${ }^{6-8} \mathrm{Ni}$ nanoparticles have been used as catalysts and the growth of homogeneous NRs has been demonstrated and could be explained based on the vapor-liquidsolid (VLS) growth model. ${ }^{9}$ Catalyst-free growth is possible under nitrogen-rich growth conditions and has been shown to lead to regular columnar growth. For that case, different growth models have been proposed. Calleja et al. have suggested that local Ga accumulation initiates GaN NR growth, and have assigned interface-near cathodoluminescence features to the presence of $\mathrm{Ga}$ interstitials as resulting defects. ${ }^{4}$ Songmuang et al. have systematically studied the nucleation and growth of GaN NRs on thin AlN wetting layers; ${ }^{8}$ in particular, they have shown that the substrate temperature is the dominating parameter to control NR growth. Debnath et $a l$. have explained the observed strong increase of NR length with decreasing diameter employing a mass transport model ${ }^{10}$ that was initially suggested by Johansson et al. ${ }^{11}$ for VLS growth of GaP NRs. It takes mass transport by diffusion on the NR sidewalls as a source for Ga adatoms into account

\footnotetext{
a) Author to whom correspondence should be addressed. Electronic mail: eickhoff@wsi.tum.de.
}

and assumes the presence of a Ga droplet on the top surface. However, direct observation of Ga droplets on the top of GaN NRs has not yet been possible.

For the realization of nano-optical or nanoelectronic devices, $n$ - and $p$-type doping of GaN NRs with silicon and magnesium is a crucial issue that has only been addressed in a few publications. ${ }^{12,13}$ Besides their impact on the optical and electronic properties, the influence of these impurities on the growth process is of importance, as they have been reported to affect the growth kinetics of $\mathrm{GaN} .{ }^{14-16}$ In addition, the simultaneous exposure of polar and nonpolar GaN surfaces during MBE growth of NRs allows us to study the impurity effects on both types of surfaces and to obtain additional insight into the NR growth process.

In the present paper, we have studied the nucleation and growth mechanism of GaN NRs grown by PAMBE under nitrogen-rich conditions on $\mathrm{Si}$ (111) substrates. Due to growth at elevated substrate temperatures, the formation of a $\mathrm{GaN}$ wetting layer was avoided. The influence of $\mathrm{Si}$ and $\mathrm{Mg}$ on the growth process is analyzed by comparing the morphology of NRs grown at different $\mathrm{Si}$ - and $\mathrm{Mg}$-fluxes.

\section{EXPERIMENTAL}

Gallium nitride nanorods (NRs) were grown by PAMBE. Nitrogen radicals were supplied by an Oxford Applied Research radio-frequency plasma source, whereas thermal effusion cells were used for $\mathrm{Ga}, \mathrm{Si}$, and $\mathrm{Mg}$. Low-resistivity $n$-type $\mathrm{Si}$ (111) substrates were etched in 5\% HF for $10 \mathrm{~s}$ to remove the surface oxide layer prior to transfer into the load lock chamber of the PAMBE system. For all samples a substrate temperature of $790{ }^{\circ} \mathrm{C}$, a nitrogen partial pressure of $2.7 \times 10^{-5}$ mbar, and a $\mathrm{Ga}$ beam equivalent pressure 

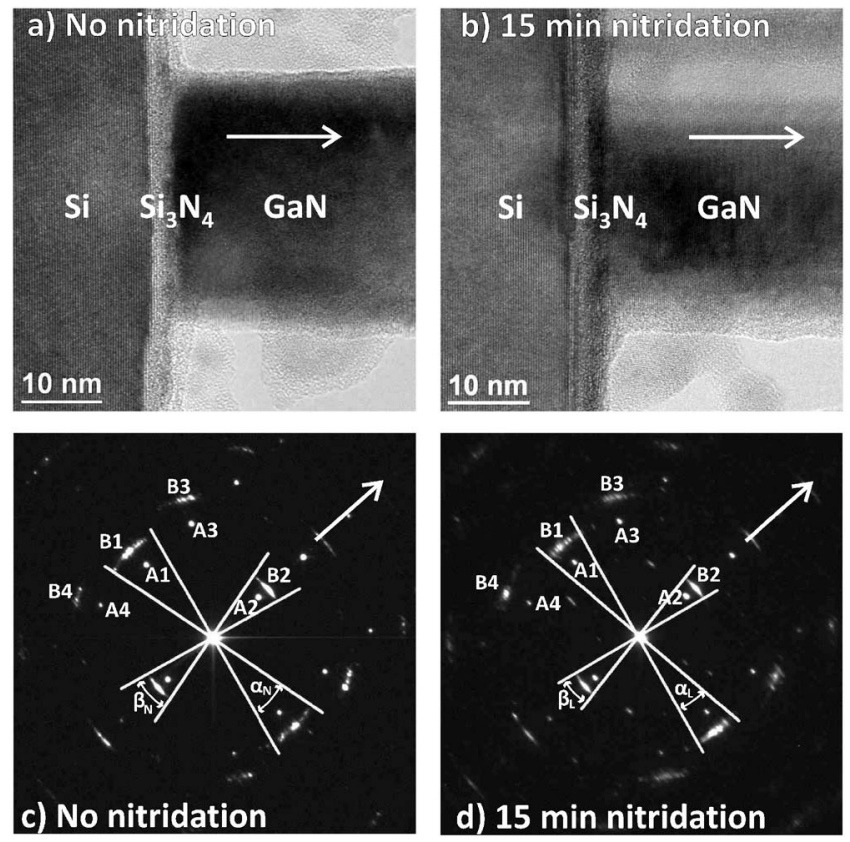

FIG. 1. (a) XTEM image of the GaN nanorod/Si (111) interface region after growth of nominally undoped nanorods without intentional nitridation. (b) XTEM image after $15 \mathrm{~min}$ additional nitridation in the initial stage of growth. Extended nitridation does not lead to a thicker silicon nitride intermediate layer but increases the homogeneity. (c) SAED pattern obtained for the sample without intentional nitridation. (d) SAED pattern of the $15 \mathrm{~min}$ nitridated sample. Arrows indicate the growth direction ( $c$ axis).

$\left(\mathrm{BEP}_{\mathrm{Ga}}\right)$ of $3.3 \times 10^{-7}$ mbar was used. The substrates were exposed to the nitrogen plasma at a temperature of $790{ }^{\circ} \mathrm{C}$ for 2 min directly before GaN growth unless mentioned otherwise. The morphology and structure were characterized by high-resolution transmission electron microscopy and scanning transmission electron microscopy (STEM) in brightfield, and high angular annular dark-field modes in a JEOL JEM2010F field emission gun microscope with a $0.19 \mathrm{~nm}$ point-to-point resolution. Electron energy loss spectroscopy (EELS) was carried out using a GIF2001 spectrometer coupled to the JEM2010F microscope. For TEM sample preparation, the GaN NRs were mechanically removed from the substrate and prepared as a suspension in n-hexane. A drop of this suspension was deposited on a holey carbon copper grid. In order to study the influence of the substrate on GaN NRs growth in more detail, cross-sectional TEM (XTEM) samples were prepared by conventional ion milling. Scanning electron microscopy (SEM) measurements in both top and cleaved edge view were performed in a Hitachi S4000n field emission SEM.

\section{RESULTS AND DISCUSSION}

\section{A. Nucleation of undoped nanorods}

XTEM measurements reveal that prior to nucleation of the NRs a thin amorphous silicon nitride layer with a thickness between 1.5 and $2 \mathrm{~nm}$ is formed on the Si (111) surface. In Fig. 1, XTEM micrographs of the bottom part of a single NR [Figs. 1(a) and 1(b)] and selected area electron diffraction (SAED) patterns [Figs. 1(c) and 1(d)] for samples with nitridation times of 0 and $15 \mathrm{~min}$ are shown. Growth axes are
TABLE I. Indexation of the SAED patterns for the nanorod samples shown

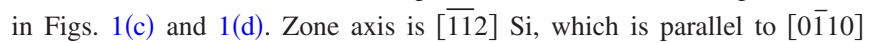
$\mathrm{GaN}$.

\begin{tabular}{ccc}
\hline \hline Spot & ${ }^{\circ}$ vs. Spot $\mathrm{A}_{1}$ & Indexation \\
\hline $\mathrm{A}_{1}$ & $\ldots$ & $(2 \overline{2} 0) \mathrm{Si}$ \\
$\mathrm{A}_{2}$ & 90 & $(111) \mathrm{Si}$ \\
$\mathrm{A}_{3}$ & 31 & $(3 \overline{1}) \mathrm{Si}$ \\
$\mathrm{A}_{4}$ & 31 & $(1 \overline{31}) \mathrm{Si}$ \\
$\mathrm{B}_{1}$ & 0 & $(\overline{2} 110) \mathrm{GaN}$ \\
$\mathrm{B}_{2}$ & 90 & $(0002) \mathrm{GaN}$ \\
$\mathrm{B}_{3}$ & 32 & $(\overline{2} 112) \mathrm{GaN}$ \\
$\mathrm{B}_{4}$ & 32 & $(\overline{2} 11 \overline{2}) \mathrm{GaN}$ \\
\hline \hline
\end{tabular}

marked with arrows in both XTEM images and SAED patterns. In Table I we show the indexation of the SAED patterns, which is equivalent for both samples. The epitaxial relationship of the GaN NRs with respect to the $\mathrm{Si}$ (111) surface was found to be (0002)[0 $\overline{1} 10] \mathrm{GaN} \|(111)[\overline{11} 2] \mathrm{Si}$. In addition, convergent beam electron diffraction (CBED) revealed that the growth direction is (0001), i.e., the NRs exhibit Ga-face polarity. The respective experimental and simulated CBED patterns are shown in Figs. 2(a) and 2(b). ${ }^{17,18}$ Most of the GaN NRs keep an epitaxial relationship with the $\mathrm{Si}$ substrate. However, some of the NRs present a certain tilt with respect to the normal direction, as indicated by the presence of faint arcs around the (0002) and (2110) main spots in Figs. 1(c) and 1(d). The maximum tilt angle has been calculated for $(\overline{2} 110)(\alpha)$ and (0002) $(\beta)$ planes, obtaining $\alpha_{N}$ $=26^{\circ}$ and $\beta_{N}=27^{\circ}$ for the non-nitridated sample, and $\alpha_{L}$ $=20^{\circ}$ and $\beta_{L}=21^{\circ}$ for a nitridation time of $15 \mathrm{~min}$. These results can explain the differences found between both samples: In general, we observe more homogeneous growth of NRs with reduced tilting angles for longer nitridation times, i.e., a beneficial role of the surface nitridation. An inhomogeneous silicon nitride layer might also be responsible for the inhomogeneous orientation of GaN NRs reported by Songmuang et al. in Ref. 8. The silicon nitride layer is formed in a self-passivating manner during the initial stage of NR growth, when the heated substrate is exposed to the nitrogen plasma. Extended nitridation results in a more homogeneous $\mathrm{Si}_{3} \mathrm{~N}_{4}$ layer rather than an increase of its thickness. From these results it is evident that NR growth and the related surface processes take place on a silicon nitride surface. For the growth temperature applied here we do not observe the formation of a $\mathrm{GaN}$ wetting layer in contrast to earlier works, ${ }^{6,7}$ indicating that the sticking coefficient for $\mathrm{Ga}$ adatoms on the nitridated $\mathrm{Si}$ (111) surface is low. This be-
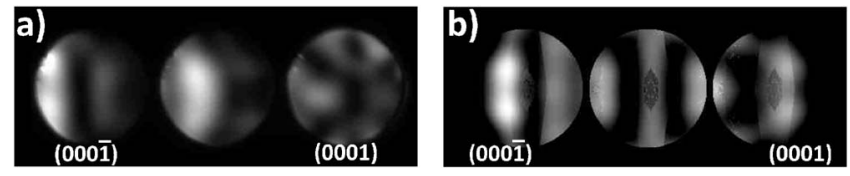

FIG. 2. (a) CBED pattern obtained in sample with a 2 min nitridated sample. (b) Simulated CBED pattern confirming that the GaN growth occurs along the (0001) direction (Ga-face polarity). 

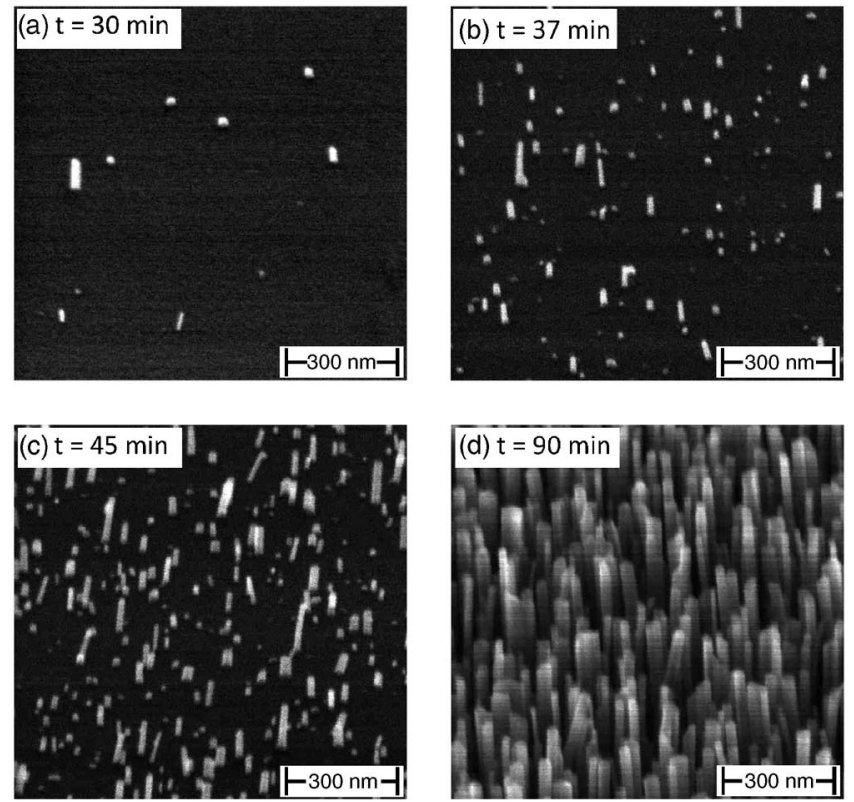

FIG. 3. SEM images of GaN nanorod samples grown on a Si (111) substrate after different growth durations, taken under a declination angle of $45^{\circ}$ Nucleated nanorods were observed for growth times above $30 \mathrm{~min}$. Nucleation proceeded up to a growth time of $60 \mathrm{~min}$. After that, the density of nanorods decreased due to merging and coalescence.

havior was also described in Ref. 19, where Si (111) surfaces are used as pseudomasks for selective area growth of GaN by ammonia MBE.

To further investigate the nucleation process, the nucleation density as a function of growth time was analyzed by top-view SEM imaging. After $30 \mathrm{~min}$ a low rod density of approximately $5 \mathrm{NR} / \mu \mathrm{m}^{2}$ is found, shown in the SEM micrograph in Fig. 3(a). If the growth time is increased, a strong increase of the NR density from $85 \mathrm{NR} / \mu \mathrm{m}^{2}$ at 37 $\min$ [Fig. 3(b)] to $150 \mathrm{NR} / \mu \mathrm{m}^{2}$ [Fig. 3(c)] at $45 \mathrm{~min}$ and to $450 \mathrm{NR} / \mu \mathrm{m}^{2}$ at $60 \mathrm{~min}$ is found, followed by a decrease to an apparent value of $275 \mathrm{NR} / \mu \mathrm{m}^{2}$ at $90 \mathrm{~min}$ [Fig. 3(d)] and $75 \mathrm{NR} / \mu \mathrm{m}^{2}$ at $300 \mathrm{~min}$, the latter being caused by increasing coalescence of neighboring NRs as growth proceeds. In Fig. 4, the measured nucleation density is shown as a function of growth time for a substrate temperature of $790{ }^{\circ} \mathrm{C}$ (solid triangles). The nucleation time $t_{\text {nucl }}$, which represents the sudden increase of the nucleation density, sensitively depends on the substrate temperature. As shown in Fig. 4, a slight decrease of the substrate temperature by approximately $10{ }^{\circ} \mathrm{C}$ (realized by investigating the edges of the $1 \times 1$ in. substrate, which are closer to the tantalum substrate holder) leads to a decrease of the nucleation time (open triangles). An increase of the substrate temperature further delays the nucleation, as shown by the data point for a substrate temperature of $T_{\text {sub }}=795{ }^{\circ} \mathrm{C}$ (open circle) at $45 \mathrm{~min}$. For $T_{\text {sub }}$ $=790{ }^{\circ} \mathrm{C}$, a maximum nucleation density of approximately $450 \mathrm{NRs} / \mu \mathrm{m}^{2}$ was found for a growth duration of $60 \mathrm{~min}$. The apparent decrease for longer growth times is due to the merging of slightly tilted NRs with adjacent NRs oriented perpendicular to the substrate surface by large-angle coalescence, as shown in the TEM image of Fig. 4(b). It should be mentioned that the density of tilted NRs and thus the resulting density of joints is reduced in the case of Si-doped NRs.

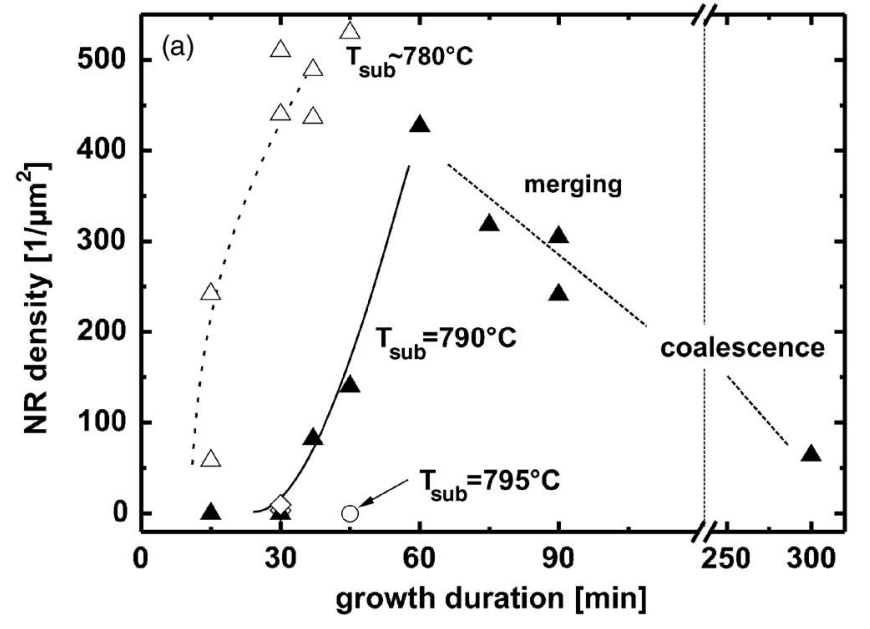

(b)

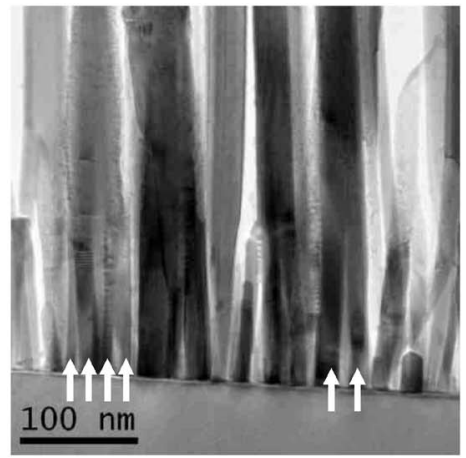

FIG. 4. (a) Nanorod density as a function of growth time. For a substrate temperature of $790{ }^{\circ} \mathrm{C}$ (solid triangles) nucleation is finished after approximately $60 \mathrm{~min}$. A decrease in substrate temperature leads to a decreased nucleation time (open triangles), whereas an increase leads to no apparent nucleation even after $45 \mathrm{~min}$ of growth (open circle). For a substrate temperature of $790{ }^{\circ} \mathrm{C}$, two different additional nitridation times $(0,45 \mathrm{~min})$ besides the standard nitridation time of $15 \mathrm{~min}$ were evaluated after $30 \mathrm{~min}$ growth, yielding almost identical values. (b) Bright-field scanning TEM image showing the merging of tilted and adjacent straight nanorods, leading to an apparent decrease of the nanorod density.

For long growth times, partial coalescence of neighboring NRs occurs and causes a further decrease of the NR density, also indicated in Fig. 4(b). In addition, we have observed that an increase of the nitridation does not accelerate the nucleation process, demonstrated by the same nucleation density of samples with no additional nitridation and additional nitridation times of 15 and $45 \mathrm{~min}$, measured at a growth time of $30 \mathrm{~min}$ [Fig. 4(a)]. The long nucleation times observed here cannot be explained by "Ga-balling" as the responsible nucleation mechanism, suggested in Ref. 4. Instead, we consider the initial nitridation of the Si (111) surface as responsible for the creation of nucleation sites under the growth conditions applied here. During the nitridation process, nitrogen atoms penetrate the silicon substrate. Due to the longer $\mathrm{Si}-\mathrm{N}$ bond compared to the $\mathrm{Si}-\mathrm{Si}$ bond, this is accompanied by the generation of local compressive strain fields. In addition, interstitial silicon atoms are generated that can diffuse to the surface, enhancing local nucleation of GaN. Indeed, we have observed the diffusion of $\mathrm{Si}$ into the GaN NR by spatial resolved electron energy loss spectroscopy (EELS) measurements, displayed in Fig. 5. Whereas the Si signal is detectable up to several nanometers in the GaN NR, the Ga 


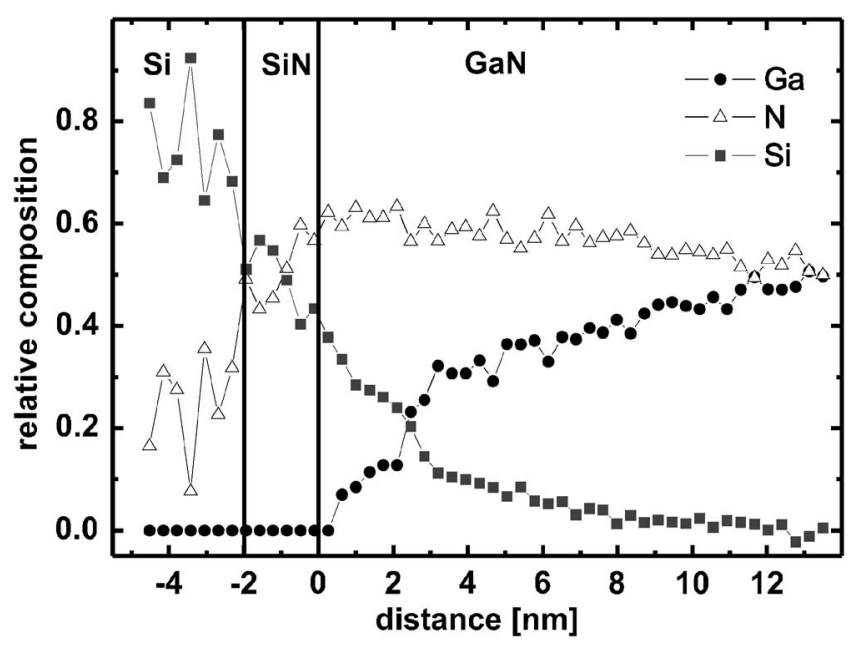

FIG. 5. Spatially resolved EELS measurement of the GaN/Si interface region. Besides the formation of a silicon nitride interfacial layer, diffusion of silicon into the $\mathrm{GaN}$ nanorod is observed.

signal completely disappears in the silicon nitride layer, demonstrating that the observed result is no effect of beam dispersion on the sample. The formation of $\mathrm{GaN}$ islands in the initial stage of NR growth on AlN nucleation layers has also been reported in Ref. 8.

Summarizing the results presented above, we have found that the growth conditions applied here result in long nucleation times of GaN NRs ( $>30 \mathrm{~min})$ and inhibit the formation of a wetting layer, in contrast to earlier reports. ${ }^{4,7} \mathrm{We}$ attribute this behavior to the higher growth temperature. This conclusion is strongly supported by the similarity of Fig. 3(b) in this work and Fig. 4b of Ref. 6. Compared to the standard NR samples in that reference, the latter one was grown at substrate temperatures approximately $20{ }^{\circ} \mathrm{C}$ higher. In the present study, the nucleation time could be decreased by reduction of the substrate temperature, leading to similar results as in Ref. 6. At higher substrate temperatures, with no $\mathrm{GaN}$ wetting layer being present, local strain fields or silicon rich areas as well as steps on the nitridated $\mathrm{Si}$ (111) surface can act as nucleation sites for the formation of $\mathrm{GaN}$ islands. On a Si (111) surface, the high substrate temperatures cause an increase in nucleation time. Comparison to the results in Ref. 8 shows that this latter effect can be attributed to a high adatom diffusivity on the nitridated $\mathrm{Si}$ (111) surface, as growth on an AlN buffer layer was shown to lead to immediate nucleation.

\section{B. Growth of undoped nanorods}

The dependence of the NR length on the growth time is shown in Fig. 6(a). The NR length cannot be measured for growth times shorter than $t_{\text {nucl }}$. After the nucleation phase we find a constant growth rate of $(8.5 \pm 2.2) \mathrm{nm} / \mathrm{min}$ up to a NR length of $2.4 \mu \mathrm{m}$, as indicated by the solid line. The dependence of the NR diameter on the growth duration is shown in Fig. 6(b), as deduced from top-view SEM analysis. As a general trend the diameter increases with increasing growth time, although the determination of a radial growth rate is not possible from the displayed data: For growth times below 60 min the NR diameter is constant. The apparent
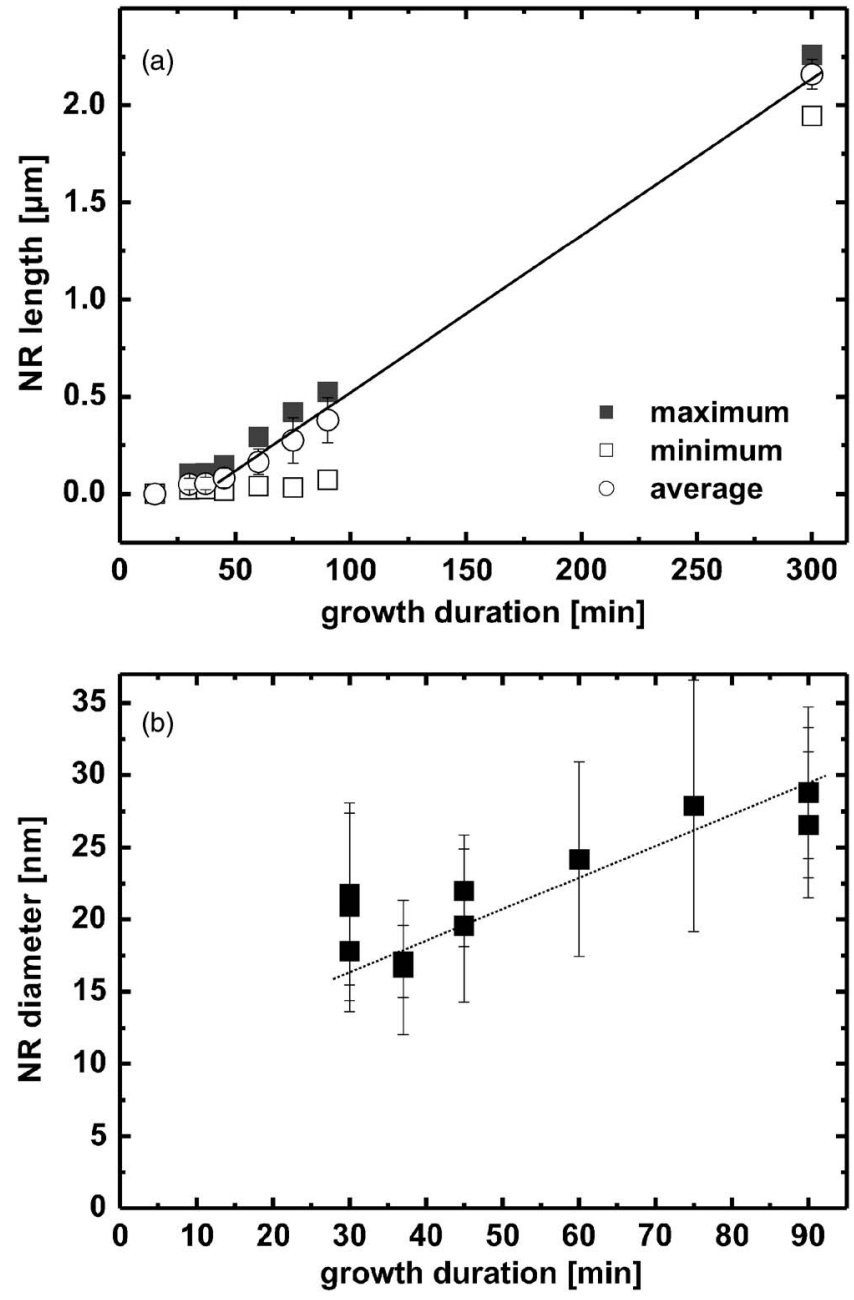

FIG. 6. (a) Dependence of the nanorod height on the growth duration. After nucleation, a constant growth rate of $(8.5 \pm 2.2) \mathrm{nm} / \mathrm{min}$ is obtained. (b) Dependence of the nanorod diameter on the growth duration. A real radial growth rate cannot be extracted as the apparent increase of the diameter at $t=60 \mathrm{~min}$ is due to the merging of adjacent nanorods [cf. Fig. 4(b)].

increase for longer growth durations can be attributed to the merging and coalescence of neighboring NRs according to Fig. 4(a).

Consequently, the slope of a linear fit to the data in Fig. 6(b) can be considered as the upper limit for the radial growth rate, and is $1.7 \AA / \mathrm{min}$. These results differ significantly from earlier reports on catalyst-free NR growth by PAMBE. In particular, we have not observed the strong dependence of the NR length on the diameter, as it was reported in Ref. 10. On the contrary, the variation of NR lengths observed here is due to different nucleation times for individual NRs [cf. Fig. 3(a)] and remains almost constant during the rest of the growth process. We attribute this different behavior to the higher growth temperature applied in the present work: The formation of a GaN wetting layer is suppressed, thereby causing a higher diffusivity of Ga adatoms on the nitridated silicon (111) surface. Due to the high density of NRs, proximity effects with respect to competitive incorporation of $\mathrm{Ga}$ adatoms in adjacent rods cannot be neglected. The constant axial growth rate for a NR length up to $2.5 \mu \mathrm{m}$ [cf. Fig. 6(a)] and the regular prismatic NR shape suggests that, due to a high diffusion length on nonpolar 

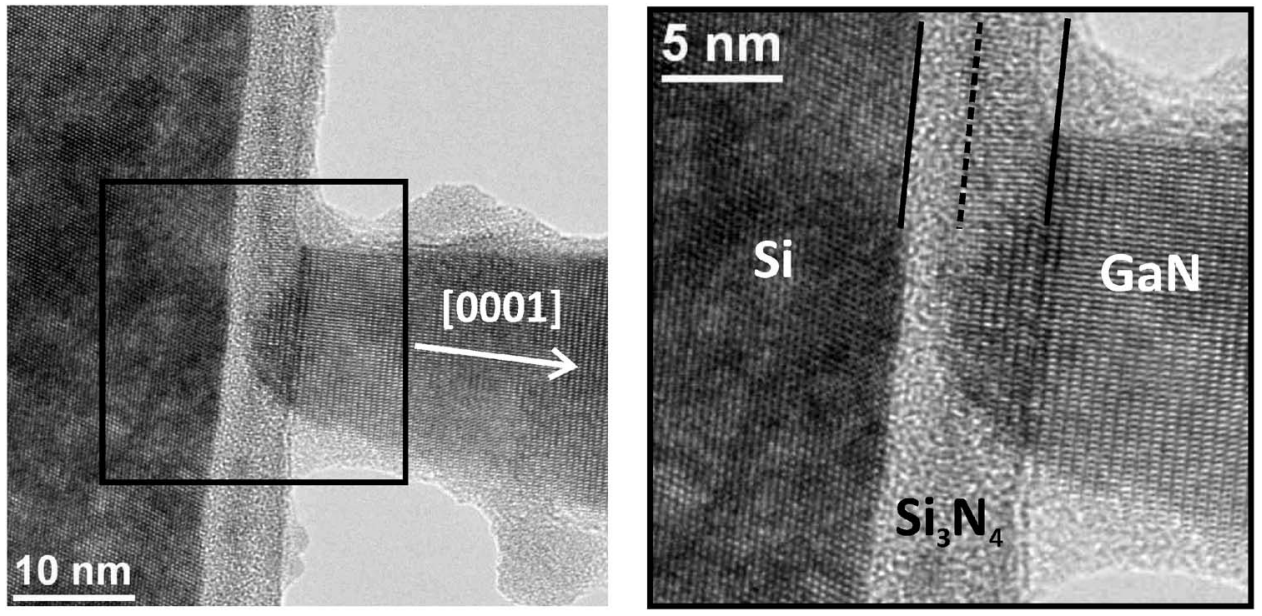

FIG. 7. XTEM image of the GaN nanorod/Si (111) interface region of a highly silicon doped $\mathrm{GaN}$ nanorod with $\mathrm{BEP}_{\mathrm{Si}}=7 \times 10^{-10}$ mbar. The formation of an additional silicon nitride layer after nucleation of the nanorod is observed between the nanorods. The amorphous material on the sidewalls of the NR is part of the glue used for XTEM sample preparation.

surfaces, Ga adatoms diffuse along the NR sidewalls and are incorporated on the top surface. These findings agree with the results reported in Ref. 8, where GaN NRs on an AIN buffer layer, grown at a substrate temperature of $790{ }^{\circ} \mathrm{C}$, showed a constant axial and almost no radial growth rate once the nucleation phase was finished. Also, adatom diffusion on the whole length of NR sidewalls was reported. In Ref. 9, where GaN NR growth is initiated by Ni nanoparticles, unperturbed diffusion along the sidewalls has been observed up to a NR length of $7.5 \mu \mathrm{m}$. In contrast, a diffusion length of approximately $40 \mathrm{~nm}$ on the NR sidewalls is extracted from the dependence of the NR length on the diameter in Ref. 10, where a lower growth temperature was applied. In the following, the influence of $\mathrm{Si}$ and $\mathrm{Mg}$ doping on the growth process of GaN NR will be investigated. Both impurities have been reported to affect the adatom kinetics on GaN surfaces. ${ }^{14-16}$

\section{Influence of silicon}

The presence of silicon is known to influence the surface kinetics during GaN growth. Theoretical investigations revealed that under nitrogen-rich and silicon-rich growth conditions GaN growth on $\mathrm{GaN}$ (0001) surfaces is unstable against the formation of $\mathrm{Si}_{3} \mathrm{~N}_{4}$ due to $\mathrm{Si}$-induced surface reconstructions. ${ }^{14}$ Figure 7 shows a XTEM micrograph of the bottom part of a highly Si-doped $\mathrm{GaN}$ NR $\left(\mathrm{BEP}_{\mathrm{Si}}=7\right.$ $\times 10^{-10}$ mbar).

As for the nominally undoped NRs, a silicon-nitride layer with a thickness between 1.5 and $2 \mathrm{~nm}$ is formed in the initial stage of growth, visible between the NR and the Si substrate.

In addition, a second layer of silicon nitride with a thickness of approximately $3 \mathrm{~nm}$ is formed between the NRs, demonstrating that, under Si- and N-rich growth conditions, the formation of silicon nitride on the nitridated silicon surface is favored compared to the growth of GaN. The high growth-rate in the $c$-direction indicates that the adatom kinetics at the NR top surface is not significantly influenced, implying that $\mathrm{Si}$ is efficiently incorporated into the $\mathrm{GaN}$ NRs. This was independently confirmed by photoluminescence measurements. $^{20}$ TEM and XTEM measurements did not reveal any indication of the formation of silicon nitride on the nonpolar surfaces. Upon Si doping we have observed a decrease in the NR density, which we attribute to the enhancement of the amorphous silicon nitride layer, which inhibits the nucleation of GaN NRs. Simultaneously, the density of tilted NRs due to nucleation directly on the Si (111) surface is significantly reduced, which also leads to a suppression of NR merging. Further information about the influence of Si on NR growth can be extracted from Fig. 8, where the NR diameter and the NR length are displayed as a function of the $\mathrm{BEP}_{\mathrm{Si}}$ during growth. For low and medium $\mathrm{BEP}_{\mathrm{Si}}$, a continuous decrease of the NR length with increasing $\mathrm{BEP}_{\mathrm{Si}}$ is observed, accompanied by an increasing rod diameter, the latter one being caused by NR tapering as shown in the TEM images in Figs. 9(a) and 9(b). For $\mathrm{BEP}_{\mathrm{Si}}>3$ $\times 10^{-10}$ mbar, both effects are much more pronounced. TEM analysis reveals that this gradual increase of the NR diameter is caused by the occurrence of bunches of consecutive monoatomic steps along the NRs, which were observed for $\mathrm{BEP}_{\mathrm{Si}} \geq 2 \times 10^{-11}$ mbar. The distance between such step bunches decreases when the $\mathrm{BEP}_{\mathrm{Si}}$ is increased, leading to more pronounced tapering [Figs. 9(a) and 9(b)]. The mechanism leading to this step formation is not clarified yet. For a possible explanation, it has to be taken into account that a reduction of the $\mathrm{BEP}_{\mathrm{Si}}$ below $2 \times 10^{-11}$ mbar during $\mathrm{NR}$ growth results in growth continuation with a constant in-

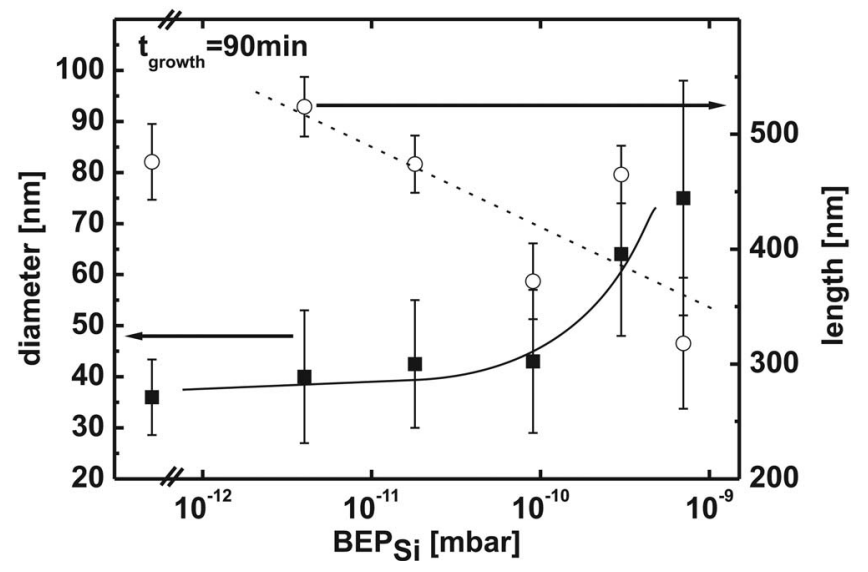

FIG. 8. Dependence of the nanorod diameter (left axis) and length (right axis) on the $\mathrm{BEP}_{\mathrm{Si}}$. An increase in diameter due to tapering of nanorods is accompanied by a decrease in height. 


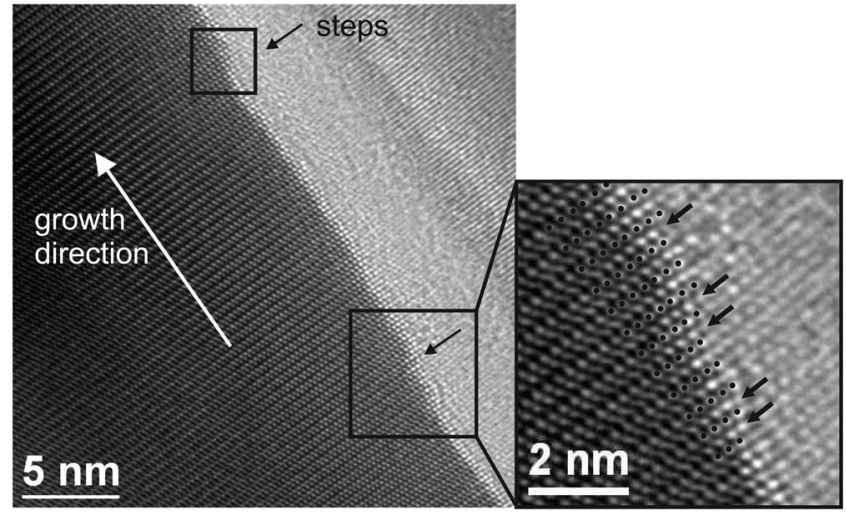

FIG. 9. High-resolution TEM image of a silicon-doped $\left(\mathrm{BEP}_{\mathrm{Si}}=2\right.$ $\times 10^{-11}$ mbar) $\mathrm{GaN}$ nanorod. The tapering effect is caused by a gradual increase of the nanorod diameter due to subsequent groups of monoatomic steps on the sidewall (indicated by the arrows). High silicon doping leads to strong tapering, a decreased nanorod density, and suppressed nanorod merging.

creased diameter (not shown). Thus, the tapering effect does not originate in the initial stage of growth but mirrors the $\mathrm{Si}$ flux during growth. As this phenomenon is accompanied by a strong decrease in the axial growth rate, we assume that it is caused by the nucleation of diffusing adatoms at steps on the nonpolar sidewalls, which are present due to the slight tilting of the NRs with respect to the growth axis, as obvious in the TEM image in Fig. 9(b). We attribute the decrease of the axial growth rate to the incorporation of diffusing $\mathrm{Ga}$ adatoms on the NR sidewalls as a competing process. This effect supports the conclusions in Refs. 8-10 that Ga adatoms diffusing along the NR sidewalls strongly contribute to the axial growth rate.

\section{Influence of magnesium}

In the case of $\mathrm{Mg}$ doping, we have also observed a decrease in the NR length and an increase of the NR diameter with increasing $\mathrm{BEP}_{\mathrm{Mg}}$, the latter being more pronounced than in the case of $\mathrm{Si}$ doping, as it is shown in Fig. 10. Compared to the case of silicon, the influence of $\mathrm{Mg}$ on the NR growth mechanism appears to be different, as it does not

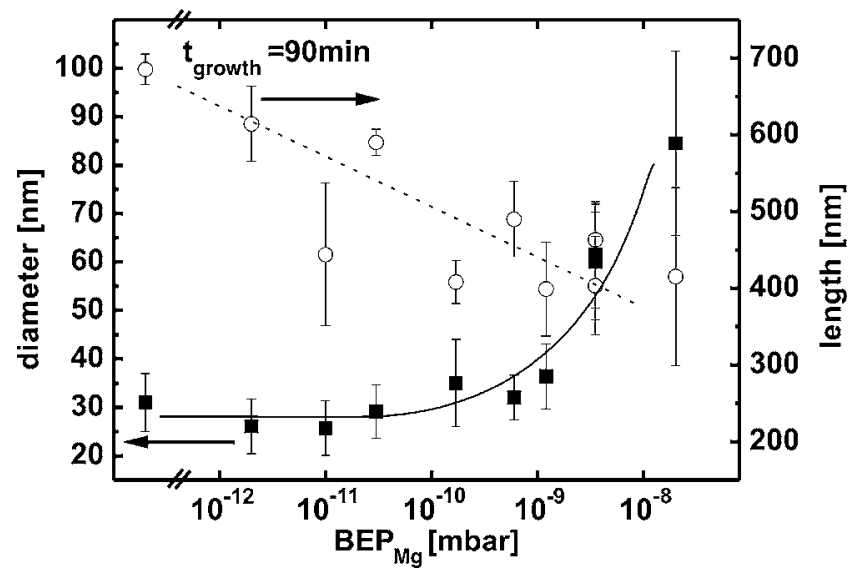

FIG. 10. Dependence of the nanorod diameter (left axis) and length (right axis) on the $\mathrm{BEP}_{\mathrm{Mg}}$. The increasing nanorod diameter is due to an increase of the lateral growth rate. Solid and dashed line are guides to the eye only.

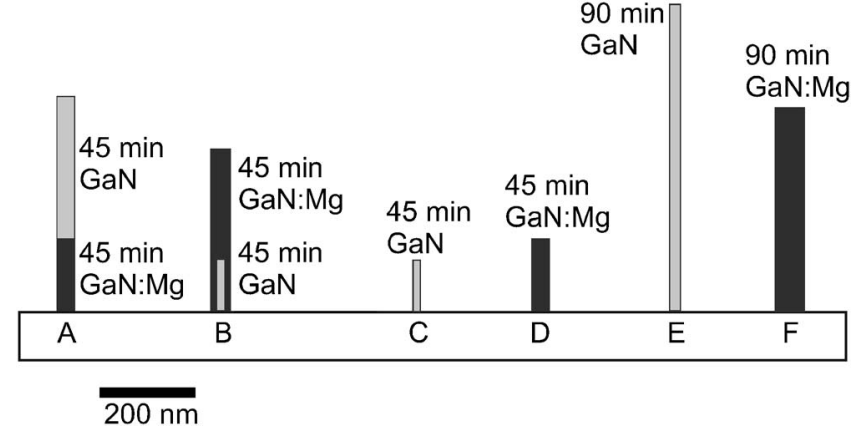

FIG. 11. Test structures to study the effect of $\mathrm{Mg}$ on nanorod growth. Schematic drawing of the samples summarized in Table II.

change the prismatic NR shape. In order to clarify whether the observed effects are caused by a change in nucleation size or by an increased radial growth rate, we have grown compound NR test structures (Fig. 11) and compared the axial and lateral dimensions. These compound structures were realized by 45 min growth of undoped $\mathrm{GaN}$ on top of a $\mathrm{Mg}$-doped NR $\left(\mathrm{BEP}_{\mathrm{Mg}}=4 \times 10^{-9} \mathrm{mbar}\right.$, growth time $45 \mathrm{~min}$, sample A) as well as a structure grown in inverted order (sample B). For comparison, undoped (C) and Mg-doped (D) reference samples were grown for 45 and $90 \min (\mathrm{E}, \mathrm{F})$, respectively. The results, summarized in Table II, allow the following conclusions: The axial growth rate is reduced due to the presence of $\mathrm{Mg}$. From samples $\mathrm{D}$ and $\mathrm{F}$ an axial growth rate of $(6.4 \pm 2.2) \mathrm{nm} / \mathrm{min}$ is calculated, compared to $(8.8 \pm 2.2) \mathrm{nm} / \mathrm{min}$ for undoped NRs [cf. Fig. 6(a)]. In contrast, comparison of $\mathrm{C}$ and $\mathrm{D}$ suggests a decreased nucleation time due to $\mathrm{Mg}$ doping. This also explains that the estimated NR volume for a fixed growth time remains constant up to a $\mathrm{BEP}_{\mathrm{Mg}}$ of $2 \times 10^{-9}$ mbar and increases for higher $\mathrm{Mg}$ fluxes.

Interesting information also can be gained from the analysis of the composite NRs: No difference could be found between the diameter of sample A, sample B, and of reference $\mathrm{D}$. This can only be explained by higher radial growth rate in the presence of $\mathrm{Mg}$ rather than by an increased nucleation diameter. The radial growth rate extracted from samples $D$ and $F$ is $(0.5 \pm 0.1) \mathrm{nm} / \mathrm{min}$, proving that the presence of $\mathrm{Mg}$ enhances growth on nonpolar surfaces. Similar results have been obtained for $\mathrm{GaN}$-growth by metal organic vapor phase epitaxy. ${ }^{21}$ This latter finding also demon-

TABLE II. Growth conditions and dimensions of reference samples and compound nanorods to analyze the effect of $\mathrm{Mg}$ doping on the growth mechanism (cf. Fig. 11).

\begin{tabular}{cccc}
\hline \hline Sample & $\begin{array}{c}\text { Growth duration } \\
{[\mathrm{min}]}\end{array}$ & $\begin{array}{c}\text { Length } \\
{[\mathrm{nm}]}\end{array}$ & $\begin{array}{c}\text { Diameter } \\
{[\mathrm{nm}]}\end{array}$ \\
\hline A (GaN on GaN:Mg) & $45+45$ & $455 \pm 50$ & $39 \pm 5$ \\
$\mathrm{~B}(\mathrm{GaN}: \mathrm{Mg}$ on GaN) & $45+45$ & $345 \pm 60$ & $41 \pm 10$ \\
$\mathrm{C}(\mathrm{GaN})$ & 45 & $110 \pm 20$ & $17 \pm 3$ \\
$\mathrm{D}(\mathrm{GaN}: \mathrm{Mg})$ & 45 & $155 \pm 35$ & $36 \pm 5$ \\
$\mathrm{E}(\mathrm{GaN})$ & 90 & $640 \pm 30$ & $23 \pm 5$ \\
$\mathrm{~F}(\mathrm{GaN}: \mathrm{Mg})$ & 90 & $435 \pm 65$ & $62 \pm 10$ \\
\hline \hline
\end{tabular}


strates that the low radial growth rate of GaN NRs is also due to the different adatom kinetics on the NR sidewalls rather than being caused only by the limited availability of active nitrogen. ${ }^{8}$ The presence of $\mathrm{Mg}$ increases the probability of $\mathrm{Ga}$ adatoms to be incorporated in nonpolar surfaces.

The influence of $\mathrm{Mg}$ does not only affect the rod morphology, it also has an impact on the crystal structure. Analysis of highly Mg-doped samples by cross-sectional transmission electron microscopy (XTEM) shows the formation of twins and the presence of stacking faults also in regions which are far away from the substrate/NW interface. These results will be published in a systematic XTEM study. ${ }^{22}$

\section{CONCLUSION}

Based on the results shown above, the following mechanism for catalyst-free growth of GaN NRs by PAMBE under N-rich conditions on Si (111) surfaces is suggested: During the initial exposure of the Si (111) surface to a flow of nitrogen radicals, self-limited formation of an amorphous silicon nitride layer occurs. Under the N-rich growth conditions and at the high growth temperature applied here, Ga adatoms do not stick on this surface but exhibit a high diffusivity. Local nucleation of $\mathrm{GaN}$ is induced by local accumulation of strain or local enrichment of silicon, which outdiffuses from the substrate upon nitridation. Ga adatoms on the nonpolar NR sidewalls are not incorporated but contribute to the axial growth rate by diffusing to the top surface, exhibiting a diffusion length of more than $2.4 \mu \mathrm{m}$. The presence of Si enhances the nucleation of $\mathrm{GaN}$ on nonpolar facets, resulting in an increased NR diameter and, according to the diffusion model, to a decreased NR length. At high Si concentrations, the formation of silicon nitride occurs between the GaN NRs and reduces the nucleation density. Si-induced nucleation on nonpolar facets results in strong NR tapering. The presence of $\mathrm{Mg}$ leads to a continuous increase of the radial growth rate and, at high concentrations, to a decrease of the nucleation time. This increased radial growth rate has to be considered for the design of bipolar NR devices and suggests the $p$-type region to be grown first in order to provide access to both $n$ and $p$-type regions for the formation of electrical contacts and to avoid formation of coaxial $p n$-junctions.

\section{ACKNOWLEDGMENTS}

The authors from the Walter Schottky Institut acknowledge financial support by the Deutsche Forschungsgemeinschaft via NAWACS (Ei 518 2-1) and by the German Excellence Initiative via the Nanosystems Initiative Munich (NIM).

${ }^{1}$ Y. Huang, X. Duan, Y. Cui, and C. Lieber, Nano Lett. 2, 101 (2002).

${ }^{2}$ A. Kikuchi, M. Kawai, M. Tada, and K. Kishino, Jpn. J. Appl. Phys. 43, L1524 (2004).

${ }^{3}$ J. Ristić, C. Rivera, E. Calleja, S. Fernández-Garrido, M. Povoloskyi, and A. D. Carlo, Phys. Rev. B 72, 085330 (2005).

${ }^{4}$ E. Calleja, M. A. Sánchez-García, F. J. Sánchez, F. Calle, F. B. Naranjo, E. Muñoz, U. Jahn, and K. Ploog, Phys. Rev. B 62, 16826 (2000).

${ }^{5}$ H.-Y. Chen, H.-W. Lin, C.-H. Shen, and S. Gwo, Appl. Phys. Lett. 89, 243105 (2006).

${ }^{6}$ R. Meijers, T. Richter, R. Calarco, T. Stoica, H.-P. Bochem, M. Marso, and H. Lüth, J. Cryst. Growth 289, 381 (2006).

${ }^{7}$ K. Bertness, A. Roshko, N. Sanford, J. Barker, and A. Davydov, J. Cryst. Growth 287, 522 (2006)

${ }^{8}$ R. Songmuang, O. Landré, and B. Daudin, Appl. Phys. Lett. 91, 251902 (2007).

${ }^{9}$ L. Geelhaar, C. Chèze, W. M. Weber, R. Averbeck, H. Riechert, T. Kehagias, P. Komninou, G. P. Dimitrakopulos, and T. Karakostas, Appl. Phys. Lett. 91, 093113 (2007).

${ }^{10}$ R. K. Debnath, R. Meijers, T. Richter, T. Stoica, R. Calarco, and H. Lüth, Appl. Phys. Lett. 90, 123117 (2007).

${ }^{11}$ J. Johansson, C. Svensson, T. Mårtensson, L. Samuelson, and W. Seifert, J. Phys. Chem. B 109, 13567 (2005).

${ }^{12}$ J. E. van Nostrand, K. Averett, R. Cortez, J. Boeckl, C. Stutz, N. Sanford, A. Davydov, and J. Albrecht, J. Cryst. Growth 287, 500 (2006).

${ }^{13}$ Y. S. Park, J. H. Na, R. A. Taylor, C. M. Park, K. H. Lee, and T. W. Kang, Nanotechnology 17, 913 (2006).

${ }^{14}$ A. L. Rosa, J. Neugebauer, J. E. Northrup, C.-D. Lee, and R. M. Feenstra, Appl. Phys. Lett. 80, 2008 (2002).

${ }^{15}$ E. Monroy, T. Andreev, P. Holliger, E. Bellet-Amalric, T. Shibata, M. Tanaka, and B. Daudin, Appl. Phys. Lett. 84, 2554 (2004).

${ }^{16}$ J. Neugebauer, Phys. Status Solidi C 0, 1651 (2003).

${ }^{17}$ P. Ruterana, J. Alloys Compd. 401, 199 (2005).

${ }^{18}$ J. Komiyama, Y. Abe, S. Suzuki, and H. Nakanishi, J. Cryst. Growth 298, 223 (2007).

${ }^{19}$ H. Tang, S. Haffouz, and J. A. Bardwell, Appl. Phys. Lett. 88, 172110 (2006).

${ }^{20}$ F. Furtmayr, M. Vielemeyer, M. Stutzmann, A. Laufer, B. K. Meyer, and M. Eickhoff, J. Appl. Phys. (submitted).

${ }^{21}$ B. Beaumont, S. Haffouz, and P. Gibart, Appl. Phys. Lett. 72, 921 (1998).

${ }^{22}$ J. Arbiol, S. Estradé, F. Peirò, J. R. Morante, F. Furtmayr, M. Vielemeyer, M. Stutzmann, and M. Eickhoff (unpublished). 